\title{
CLOSED INJURIES OF THE PANCREAS \\ With Special Reference to Pseudocyst Formation
}

\author{
H. Ellis, M.Ch., F.R.C.S., and J. M. MYNORS, F.R.C.S. \\ The Radcliffe Infirmary, Oxford
}

\section{Introduction}

Lloyd, of Birmingham, in 1892 described two patients who developed intra-abdominal cysts three weeks and three months respectively after closed injuries to the abdomen. Although not the first to describe such pseudocysts of the pancreas (for example, Senn reported a case in 1885), he was original in realizing that their aetiology was an effusion of fluid from the damaged pancreas into the lesser sac.

Since that time, a number of case reports have appeared on this unusual and interesting injury which, in collected series, would appear to account for some 10-13 per cent. of pancreatic pseudocysts (Schmieden and Sebening, 1928; Bickford, 1948).

We have recently each encountered a patient with pseudocyst formation following closed abdominal trauma. A search through the records of the Radcliffe Infirmary, Oxford, yielded only five other cases of pancreatic damage in the 20-year period 1938 to $195^{8}$.

Of these seven cases of injury to the pancreas (summarized in Table I), five developed pseudocysts. A consideration of these patients, together with other published cases, reveals some interesting features of aetiology, diagnosis and management of this condition.

\section{Clinical Features}

Although an unusual complication of abdominal trauma, pancreatic injury with subsequent pseudocyst formation presents a fairly welldefined clinical picture-a moderately severe blow to the front of the flexed upper abdomen with a blunt object, immediate upper abdominal pain, which is usually not intense, a latent period varying from days to many weeks, and then rapid recrudescence of upper abdominal pain with pyrexia, tachycardia and the development of a tender swelling, often accompanied by signs of peritoneal irritation.

The subsequent course is often stormy and the mortality relatively high. A more detailed analysis of these features, point by point, reveals:

\section{(a) Type of Injury Resulting in Pancreatic Trauma}

The blow is usually of moderate severity to the front of the upper abdomen with the body flexed; in this position the abdominal muscles are relaxed and fail in their protective duties to the underlying viscera. Although the skin may be bruised, frequently there is no external sign of injury.

In the present series of pancreatic injuries three patients went over the handlebars of their bicycles or motorcycles (one definitely striking himself against the end of the handlebar in his fall), three were struck in the abdomen by blunt objects (a plank of wood, an iron bar and the steering wheel of a car involved in a collision), and one fell from a ladder.

In the published reports of 40 patients with closed pancreatic injury where accurate details of the type of accident were given many had definitely localized blunt injuries to the abdomen. These are summarized in Table II.

\section{(b) History and Physical Signs of Traumatic Pseudocyst Formation}

Upper abdominal pain usually develops immediately but may be delayed; in Case $I$ in the

\section{TABLE 2}

Types of Accident in 40 Cases of Pancreatic Injury Collected from the Literature

$\begin{array}{llll}\text { Kick or punch on abdomen } & \text {. } & \text {. . } & 14\end{array}$

Involved in vehicle collision $\quad . . \quad \ldots .12$

Struck on front of abdomen by blunt $\begin{array}{lllllll}\text { object } & \ldots & \ldots & \ldots & \ldots & \ldots & 6 *\end{array}$

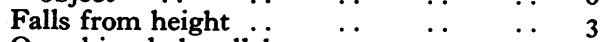

$\begin{array}{lllll}\text { Over bicycle handlebars } & \ldots & \ldots & \ldots & 2\end{array}$

$\begin{array}{llllll}\text { Crush injury } & \ldots & \ldots & \ldots & \ldots & 2\end{array}$

Trampled by horse $\quad \ldots \quad \ldots . \quad \ldots \quad$ r

40

*Edge of lorry 2, wheel of car, baseball, iron post and edge of bench. 


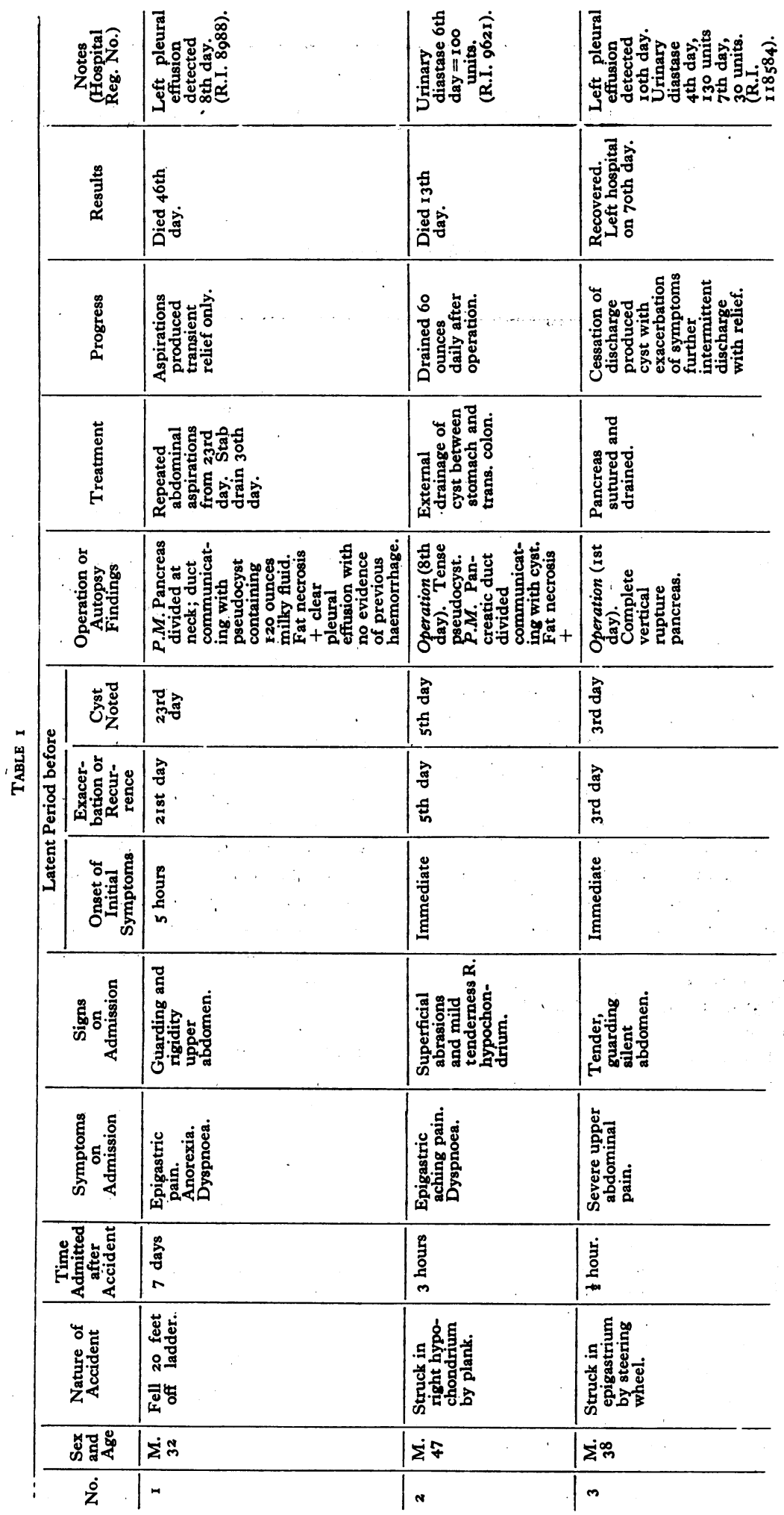

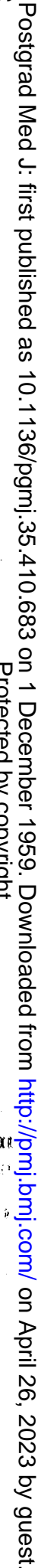




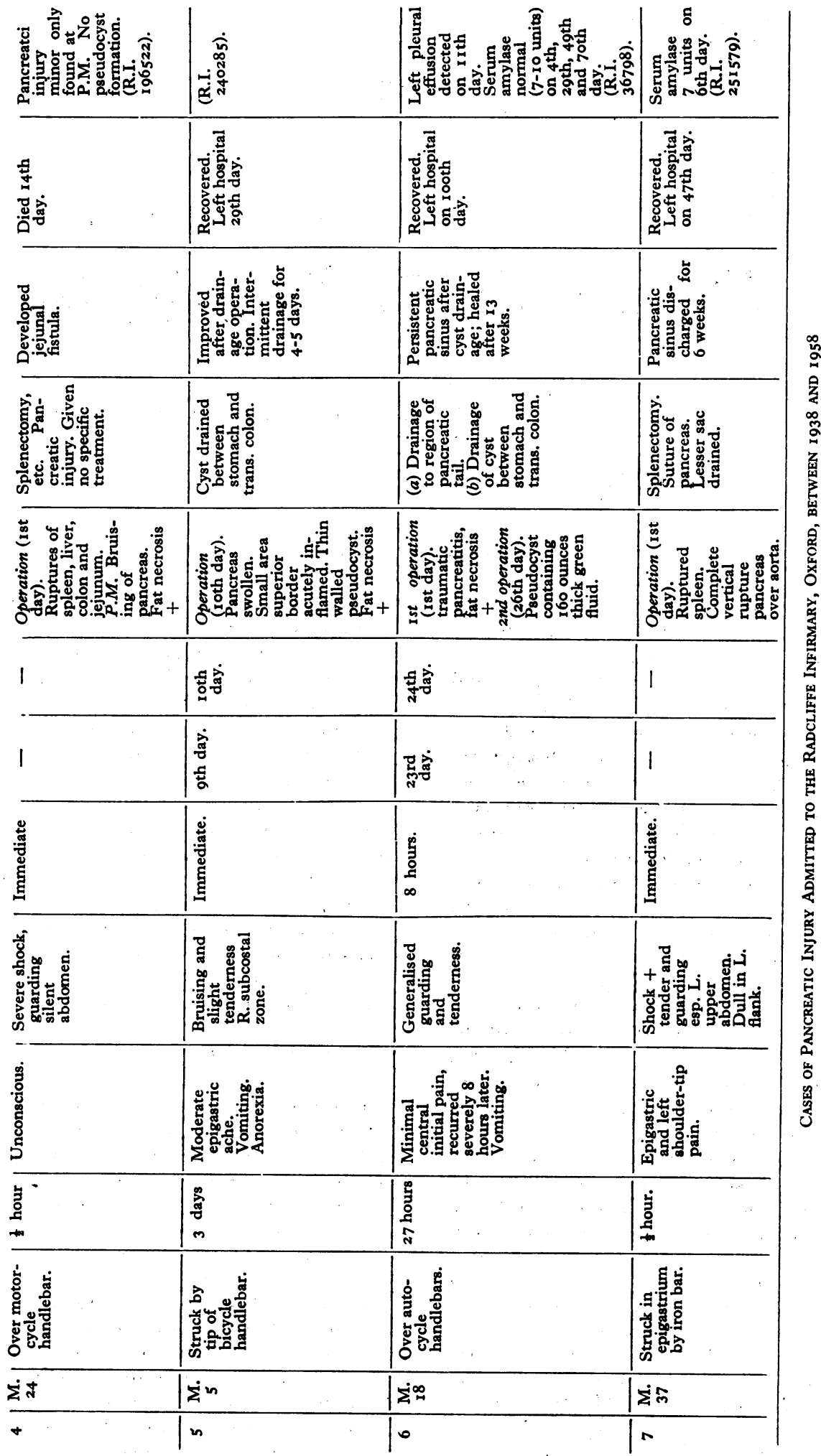

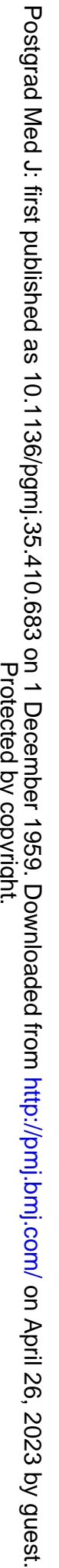




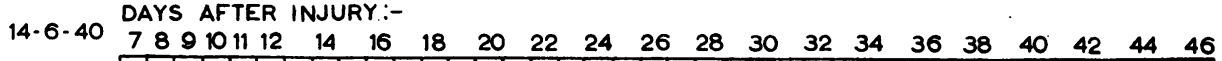

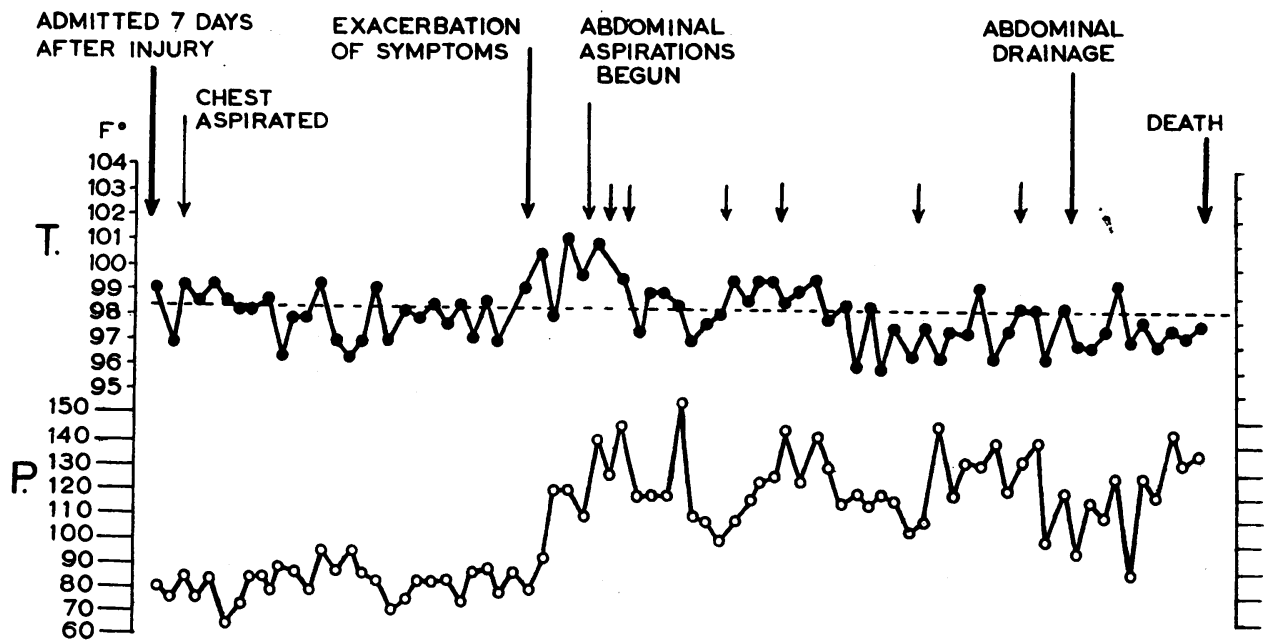

Fig. 1.- Temperature-pulse chart of Case I.

present series it was noted five hours after the accident. In uncomplicated cases it is often of only moderate severity; Cases $I$ and 5 were admitted to hospital several days after injury; and Case 2 continued work for the rest of the day.

A latent period then commonly occurs during which the pain decreases or entirely disappears; this is followed by rapid development of more severe epigastric pain accompanied by associated symptoms which may include malaise, anorexia, vomiting, dyspnoea and epigastric fullness.

In this series the latent period ranges from three to 23 days. Aldis (1946) gives an average of four to six weeks before cyst formation occurs, and much longer periods have been recorded. Meyer, Sheridan and Murphy (1949), for example, quote one case with an interval of four years from the time of injury until the discovery of the cyst. However, in such cases one suspects that the fluid collection had, in fact, remained undetected for a long period, or indeed, that the cyst was not traumatic in origin.

In those patients with injury confined to the pancreas, initial physical signs are often minimal (Case 5 for example was examined carefully in the casualty department only one hour after injury and there was complete absence of abnormal abdominal physical signs). However, there may be a little bruising of the upper abdomen with local tenderness shortly after the accident.

When pain recurs, there are marked signs of peritoneal irritation due to traumatic pancreatitis. This is well shown by Case 6 who was explored at this stage some 30 hours after injury; the typical features of a darkly bloodstained intra- peritoneal effusion, a bruised, swollen pancreas 옥 and spots of fat necrosis were discovered.

Pseudocysts in this series were detected an $\$$ indeed, appeared to have collected, in from $3 \frac{\mathbb{Q}}{2}$ to 48 hours after this recurrence of pain; thei development was associated typically with a risin $\mathbb{\Phi}$ and swinging pyrexia up to $\mathrm{IOI}^{\circ} \mathrm{F}$. and ag increase in pulse rate (Fig. I).

The cyst itself presents as a tense tender swelle ing over which the normal abdominal resonance to percussion is usually impaired. Peritoneal irritation, with its associated muscle guarding, often prevents accurate delineation of the boundaries of the mass which, in four of the five cases here recorded, extended from the xiphoid to the region of the umbilicus and, in the fifth, occupied most of the abd omen.

\section{Associated Injuries}

As the pancreas lies on the posterior wall of the lesser sac, one would expect injury to this organ by blunt trauma to be associated with extensive damage to other viscera. In the present study, Case 7 was, in fact, complicated by rupture of both the spleen and liver, and Case 4 had numerous intra-abdominal injuries of which that to the pancreas was least important and only a post-mortem finding.

However, both in our own cases and in the literature, it is far commoner for the pancreas to be the only intra-abdominal organ to be injured. We have already noted that it tends to be damaged by direct central anterior violence with the abdominal muscles flexed and therefore relaxed. The pancreas, in its relatively fixed retro-peritoneal 
position, is presumably trapped between the blow and the vertebral column; the more mobile intraperitoneal organs are usually able to 'float' away and escape injury.

It is interesting that three patients (Cases $I$, 3 and 6) developed a sterile left-sided pleural effusion which, in one, was bloodstained. Bickford (1948) has reported two examples of pleural effusion (one initially bloodstained) associated with traumatic pseudocyst of the pancreas which, again, were both on the left side. He concluded that both were probably due to concomitant chest injury, in spite of no direct evidence of this. In our own patients there was neither clinical nor radiological evidence of chest injury and we suggest that these effusions were secondary to pleural irritation by fluid in the lesser sac where this comes into direct relation to the left diaphragm.

In this context one notes that Traquair (1946) has recorded the development of a left-sided chylothorax three months after the drainage of a pancreatic pseudocyst which, in turn, presented six weeks after a kick in the epigastrium. Six months after the injury the patient developed chylous ascites. No explanation was given for these phenomena.

\section{Special Investigations}

\section{Serum and Urinary Amylase (Diastase)}

Damage to the pancreatic acini liberates amylase into the interstitial tissues or peritoneal cavity whence it is absorbed into the blood stream and rapidly excreted into the urine. The concentration of amylase in the serum is expressed either in Wohlgemuth or Somogyi units; the Wohlgemuth units give a normal range of 3 to ro units per $\mathrm{ml}$. in serum and of 2 to 50 units per $\mathrm{ml}$. in urine. The Somogyi normal range is 60 to $180 \mathrm{mg}$. reducing sugar in $100 \mathrm{ml}$. of serum. This difference in range between the two methods should be carefully noted or confusion may easily arise.

A raised amylase level following abdominal trauma has been emphasized as an important factor in diagnosing injury to the pancreas (Naffziger and McCorkle, 1943; Shallow and Wagner, 1947). In the present series the serum amylase was estimated in two patients and was normal in both. Moreover, in neither of the two cases where urinary amylase estimations were performed was the level raised significantly.

The fall in level of the amylase occurs rapidly after its initial rise in pancreatitis and it may well be that the optimum time was missed in this series. The rarity of traumatic pancreatitis is such that amylase estimations tend to be performed late on in the course of the illness (on the fourth to eighth day in this series).

\section{Blood Count}

A mild, persistent neutrophil leucocytosis, with a total white cell count of the range $12,000-14,000$, is usually present, too low a figure to suggest a large abscess with which the mass may be confused.

\section{Abdominal Radiography}

Plain abdominal radiograms (carried out in three of the present series) are not helpful. A barium meal examination may reveal displacement of the stomach and small intestine by the tumour, but was not carried out on any of the patients in this study.

\section{Examination of Cyst-aspirate}

Should the cyst be aspirated (as in three of the present series) a serous or haemorrhagic fluid will be obtained which is alkaline in reaction with an amylase cuntent ranging from 250 to 2,000 Wohlgemuth units per ml.

\section{Treatment}

If laparotomy is carried out at the stage of initial trauma, careful search must also be made for damage to other viscera. Laceration of the pancreas is dealt with by superficial sutures passed with care to avoid the main pancreatic duct. If the pancreas is completely divided, a post-operative fistula will almost certainly form, although this may heal spontaneously (Case 7 in this series); if the division is through the tail, as sometimes occurs, for example, with splenic rupture, primary excision of the distal portion is indicated in order to avoid subsequent fistula formation.

In all cases, free drainage of the lesser sac must be established in as direct a line as possible, preferably between the stomach and transverse colon, the abdominal wall being carefully protected from digestion by immediate treatment with 'Nobecutane' or Silicone barrier cream.

In the treatment of the established posttraumatic pseudocyst, the possible methods are: Repeated aspiration, drainage, marsupialization, anastomosis to bowel and excision. Complete excision is impossible; aspiration, although sometimes of diagnostic value, gives little or no relief. Anastomosis is of value in those pseudocysts which have a tough capsule which will hold sutures well; stomach, duodenum and gallbladder have all been used, but jejunum is probably the most suitable viscus into which the cyst - should be drained (Chesterman, 1943). Unfortunately, the capsule wall of the traumatic pseudocyst is usually thin and friable.so that in the vast majority of cases adequate drainage or marsupialization is, in fact, the only method 
available; following this, a fistula for a period of many weeks must be anticipated. Treatment includes meticulous care of the surrounding skin, suction and maintenance of the patient's general nutrition and electrolyte balance. Providing the main duct of the pancreas is intact or its approximation has been reasonably accurate, the majority will eventually heal, although, on occasions, transplantation of the fistula track into the bowel, or excision of the fistula together with the injured distal part of the pancreas may be required.

\section{Summary}

Seven examples of closed pancreatic injury have been studied. One was a relatively minor post-mortem finding in a patient who had died of extensive injuries; five of the remaining six proceeded to pseudocyst formation.

The clinical features and management of this type of injury are discussed.

Treatment comprises suture and drainage of the initial pancreatic tear. If pseudocyst formation occurs, drainage is usually the only possible treatment.

\section{Acknowledgments}

We would like to thank the surgical staff of the Radcliffe Infirmary for permission to publish these case reports of patients under their care, and especially Mr. D. C. Corry, Mr. A. S. Till and Dr. A. H. T. Robb Smith for helpful criticism and advice.

\section{Addendum}

Since writing this paper one of the authors has operated on a further patient with contusion of the pancreas.

Repeated estimations of the serum and urinary amylase were normal during the first 24 hours following the injury.

\section{BIBLIOGRAPHY}

ALDIS, A. S. (1946), Brit. F. Surg., 33, 323.

BICKFORD, B. J. (1948), Brit. med. $\mathcal{F}$., i, 1134 .

CHESTERMAN, J. T. (1943), Brit. F. Surg., 30, 234.

LLOYD, J. (1892), Brit. med. F., ii, ro51.

MEYER, K. A., SHERIDAN, A. I., and MURPHY, R. F. (1949), Surg. Gynec. Obstet., 88, 219.

NAFFZIGER, H. C., and McCORKLE, H. J. (1943), Ann. Surg., I18, 594 .

SCHMIEDEN, V., and SEBENING, W. (1928), Surg. Gynec. Obstet., 46, 735 .

SENN, N. (1885), Quoted by Lloyd.

SHALLOW, T. A., and WAGNER, F. B. (1947), Ann. Surg. $125,66$.

TRAQUAIR, K. (1946), Brit. F. Surg., 33, 297.

\section{in Preventive Medicine}

The prominent part played by diet in preventive medicine is now fully recognised. A well-balanced diet can contribute markedly to general good health and, conversely, a faulty diet can precipitate illness and hinder recovery. Marmite is a useful protective food providing the important B vitamins in a convenient and appetising form.

\section{for Pregnant Women}

During pregnancy the demands on all maternal resqurces are increased. Diet, therefore, assumes a particular importance during this period. Marmite, as a drink or in sandwiches, or used in cooking, is popular among expectant mothers and raises the level of the $B$ vitamins in their diet.

\section{in Geriatric Practice}

The opinion has been expressed that the need for vitamins, especially those of the B complex, is increased in frail elderly persons. These essential nutrients seem to exert a beneficial effect even when the diet appears adequate. Marmite supplies all known factors of the vitamin $B$ group; it is easily incorporated in the diet and is economical to use.

\section{in Restricted Diets}

In the dietary control of obesity, diabetes or other conditions a reduced intake of vitamins may lead to complications. Addition of Marmite to these restricted diets supplements their content of B vitamins and adds flavour to meals which are apt to be dull and uninteresting. 